\title{
MANAGEMENT OF VESICAL DYSFUNCTION OF NEUROSYPHILIS BY TRANSURETHRAL RESECTION OF THE VESICAL NECK*
}

BY

\author{
W. FOWLER
}

\author{
The Royal Hospital, Wolverhampton
}

When bladder function is permanently disorganized by syphilis of the central nervous system, measures may have to be taken for the relief of symptoms, elimination of residual urine, and prevention or eradication of urinary sepsis. In the past, there were no means by which these aims could be satisfactorily achieved. In recent years, the successful management of cord bladder by transurethral resection of the vesical neck has been reported in many papers published abroad. There is little reference to this method of treatment in the British literature, and a brief review of the published work with the results of transurethral resection of the vesical neck in three cases of cord bladder of luetic origin may be of interest.

Although it cannot be said that this method of managing cord bladder has a sound rationale, the results so far obtained support the contention upon which it is based, namely, that retention of urine in cord bladder is partly caused by obstruction at the vesical neck.

In the first place, urinary retention in cord bladder appears to result from inability of the involuntary muscle of the bladder wall, the detrusor urinae, to develop and maintain powerful contractions long enough for complete emptying of the bladder. In the autonomous and automatic bladders, this failure of detrusor contractions is due either to the bladder being isolated from a centre in the midbrain which is responsible for prolonged contractions of bladder muscle (Langworthy and Kolb, 1933), or to there being insufficient spinal segmental pathways for efficient correlation of the various detrusor impulses (Denny-Brown and Robertson, 1933b). In the atonic bladder, seen typically in tabes dorsalis, detrusor inefficiency appears to result from lack of afferent stimuli and loss of tone.

* Received for publication June 17, 1952
Because the detrusor is unable to develop and maintain powerful contractions, the internal sphincter fails to relax completely and sphincteric resistance can be considered a contributory cause of retention of urine in cord bladder. (Normally, the detrusor and internal sphincter act reciprocally, and sustained detrusor contractions are necessary for complete relaxation of the sphincter (DennyBrown and Robertson 1933a).) This reciprocal relationship persists in cord bladder (DennyBrown and Robertston, 1933b).

These primary causes of urinary retention may be supplemented by actual mechanical obstructions at the vesical neck. Thompson and Jacobson (1943) state that the degree of diverticulation present in some cases of cord bladder is indicative of mechanical obstruction at the vesical neck. These authors point out that hypertonicity and hypertrophy are characteristic features of the fully developed autonomous and automatic bladders and that these changes also affect the involuntary muscle which forms the internal sphincter at the vesical neck, and they suggest that this thickened and hypertonic sphincter may act as a barrier to the outflow of urine. Emmett (1940) and Emmett and Dunn (1946) agree with this. Nesbit and Gordon (1941) state that, as a result of proionged hypertonicity, the internal sphincter may become spastic, and they consider that this is a probable cause of retention of urine in the autonomous bladder. Definite mechanical obstruction may develop at the vesical neck when, as a result of repeated infection, the internal sphincter becomes fixed and the vesical neck contracted by scar tissue (Emmett 1940, Emmett and Dunn 1946). Emmett and Beare (1941) have drawn attention to the fact that the incidence of vesical dysfunction in tabes dorsalis is highest in those age groups in which prostatic hypertrophy is commonest, and have 
suggested that this latter condition may well add to the difficulties of micturition in tabes dorsalis.

In the light of the above it is reasonable to assume that if the resistant internal sphincter and any additional cause of obstruction at the vesical neck be removed, the incompetent detrusor may be enabled to empty the bladder more completely, although when the bladder is very atonic, the patient may still have to be taught to use other muscles to aid micturition.

The indications for the operation are usually obvious and need only be discussed in connexion with acute transverse myelitis. It will be remembered that bladder function is completely depressed for a time following the onset of this condition, and it would seem advisable that resection of the vesical neck should be delayed until such time as the bladder musculature regains tone.

The technique of the operation is a surgical concern and will not be described here. The aim is to remove as much tissue as possible from the vesical neck and also any hypertrophied or normal prostatic tissue which may be present (Emmett and Dunn, 1946). Emmett and Beare (1948) draw attention to the fact that, when tabetic cases are cystoscoped, the vesical neck may be seen to be relaxed and dilated ; they say that this should not deter the surgeon from resecting the vesical neck since the results obtained in this type of case are as good as in any other. At times, an excellent response is apparent immediately after the operation, at other times the full response may not be seen for some weeks, and Emmett and Dunn recommend an observation period of at least a few weeks before the result is finally evaluated. If the operation is judged to have failed, they state that the surgeon should have no hesitation in repeating it, as two or three "sittings" may be necessary to remove sufficient tissue for the result to be satisfactory.

Since the internal sphincter is destroyed in this operation, the state of the external sphincter (compressor urethrae) is important, for if both sphincters are functionless, all urinary control is lost. Supplied by the pudendal nerves, the external sphincter acts reflexly in conjunction with the detrusor and internal sphincter, and it is also under voluntary control in that it can be contracted at will. When the cauda equina or the conus medullaris are destroyed, it is paralysed. When the cord lesion is above the cauda equina, voluntary control of the sphincter may be lost, but it continues to act reflexly. Thus it is paralysed by lesions which produce the autonomous bladder, but continues to function when the bladder is of the automatic type. The condition of the external sphincter in tabes has been the subject of some debate. Evans (1941) states that it is paralysed, Langworthy and his colleagues (1936) believe that it may be hypertonic. As the pudendal nerves also supply the anal sphincter, the condition of this muscle will give some indication of the state of the external sphincter. Modern textbooks give little indication of the incidence and degree of paralysis of the anal sphincter in tabes dorsalis. However, Gowers (1892) states that although the anal sphincter is often weak, the paralysis rarely reaches a high degree. According to Williamson (1908), the anal sphincter may become weak in the later stages, but this is rare. Starr (1907) says that there is no relaxation of the anal sphincter. It would appear then that the external sphincter may be weak in tabes dorsalis, but is unlikely to be completely paralysed.

Emmett (1941) and Emmett and Beare (1948) have reported the results of transurethral resection of the vesical neck in 82 cases of tabes dorsalis with vesical dysfunction. Residual urine was eliminated in nearly all the 35 cases reported in 1941, and in the three cases reported in 1940. Thirty-nine of the remaining 44 cases were able to empty their bladders completely, in three cases the amount of residual urine was diminished, and only in two cases was it unchanged. Prior to the operation, thirty of the 82 cases had urinary symptoms ; these were relieved in 26, alleviated in two, and unchanged in two cases, although one of these was able to empty his bladder completely. Fifteen cases of the 1941 series were followed up by letter at least 6 years later. All the patients felt that they were emptying their bladders completely, and six reported that on examination no residual urine had been found. Nine cases had no urinary symptoms, six had varying degrees of frequency and some nocturia, and one patient returned to the clinic 7 years after operation, complaining of increasing incontinence during the previous 2 years after having been symptom-free for 5 years. He was found to have $600 \mathrm{ml}$. residual urine which was infected. After further resection of the vesical neck he was again able to empty his bladder completely. O'Leary (cited by Emmett and Beare, 1941) states that, after the vesical dysfunction had been corrected, the general condition of these patients showed a striking improvement as evidenced by gain in weight, decrease of anaemia and lassitude, and especially by decrease of frequency and of the severity of their neuritic pains.

The results of transurethral resection of the vesical neck in some 66 cases with autonomous and automatic bladders have been reported by authors already mentioned and by Thompson (1945) and 
Torres (1948). Although in many of these cases only reflex micturition was possible, the results were similar to those obtained in tabes dorsalis, residual urine being eliminated or decreased, and symptoms being alleviated in the majority of cases. Only three cases showed no improvement, and of these only one* was worse after the operation. It is rather remarkable that patients with autonomous bladders did not have continuous dribbling after the operation, but remained dry in the intervals between micturition (Nesbit and Gordon, 1941 ; Emmett and Dunn, 1946).

\section{Case Reports}

In the three cases reported below, no obvious cause of obstruction was seen at the vesical neck on cystoscopic examination and the internal sphincter was tightly closed. Although the resection was much less extensive than that advised by Emmett and his colleagues, the results were as good as those reported above.

Case 1, a male aged 59 with tabes dorsalis, was admitted to the York County Hospital on November 21, 1947. After having had N.A.B $11.3 \mathrm{~g}$. he defaulted and did not attend again until March 17, 1949, when I saw him for the first time. He had no complaints, but it was elicited that there was some delay in commencing micturition, that the urinary stream was poor, and that vesical sensation was diminished. On examination, he presented the classical signs of tabes dorsalis. After micturition, the bladder was distended half way to the umbilicus and there were $30 \mathrm{oz}$. $(885 \mathrm{ml}$.) residual urine. The urine was sterile. On rectal examination the prostate did not seem to be enlarged. He was given 10 M.U. procaine penicillin in 10 days and instructions regarding micturition. He completed the penicillin course, but did not attend the clinic again until July 14, 1949. He now complained of nocturnal incontinence and dribbling micturition. The bladder was even more distended and he was referred to Mr. Willson-Pepper who reported that there were now $44 \mathrm{oz}$. $(1,298 \mathrm{ml}$.) residual urine. On July 20, 1949 a gutter was resected in the posterior urethra extending from the internal urethral orifice to the verumontanum. Two weeks later, a bougie was passed to rupture any post-operative adhesions which might have formed. After the operation, the urinary symptoms disappeared and he was able to empty his bladder completely and with good stream. When he was last seen by me in January, 1951, micturition was still complete and easy, despite the fact that he was obviously not obeying instructions to micturate at least every 3 hours. On July 19, 1951, 2 years after the operation, he was seen again by Mr. Willson-Pepper who found no urinary symptoms and no residual urine.

Case 2, a male aged 39, was admitted to hospital in April, 1943, with retention of urine. A diagnosis of

* This patient developed incontinence (Emmett and Dunn, 1946). tabes dorsalis was made, and a permanent supra-pubic cystostomy performed. Between April, 1943, and June, 1948, he was well treated with pentavalent arsenicals and penicillin (30,000 units in aqueous suspension 3 hourly for 2 weeks). I saw him for the first time on November 10,1948 , when he complained of weakness, frequent attacks of diarrhoea and vomiting, and frequent severe attacks of lightning pains in the legs. On examination, he was thin and pale (haemoglobin 65 per cent.), and looked unhealthy. The urine was offensive and thick with pus. During the following year, attempts were made to correct the anaemia and strenuous unsuccessful efforts were made to eradicate the bladder infection. On February 8, 1950, Mr. Willson-Pepper closed the supra-pubic cystostomy and resected the posterior urethra as in the previous case. One month later, the patient was able to pass urine per urethram with a good stream and to empty his bladder completely. The only abnormalities that remained were that the call to micturition was urgent and had to be answered immediately, and that there was still some pus in the urine. Already he felt and looked much better. On May 1, 1950, he developed an acute left-sided epididymo-orchitis. The previous morning he had slipped and stretched his legs widely apart when on his way to urinate immediately after getting out of bed, and that evening had felt a pain in the left testis. The urine contained much pus, and coliform organisms were grown on culture. He was given a course of streptomycin followed by mandelates. By May 22, 1950 the epididymis and testis were normal and the urine was sterile. He was seen by me periodically until December, 1950, and during this time the urinary flow remained good, there was no residual urine, and the call to micturate was less urgent. The general improvement was similar to that described by O'Leary (cited by Emmett and Beare, 1941). He put on weight, his colour became good, the lightning pains were fewer and much less severe, and he rarely had any gastro-intestinal upset. As would be expected, his outlook on life changed very much for the better. $\mathrm{Mr}$. Willson-Pepper saw him in July, 1951, when micturition was still easy and complete, his general condition was excellent, and he was looking forward to starting work after having been unemployable for 8 years.

Case 3, a male, admitted on December 23, 1948 , complained of difficulty in commencing micturition and of constant dribbling of 1 month's duration. On examination he was euphoric, speech slightly slurred, memory good, insight very poor. There was a nodular cutaneous syphilide on the anterior aspect of both knee joints. The pupils were Argyll-Robertson, the fundi normal, and the visual fields full. The legs were spastic, the tendon reflexes of the legs greatly exaggerated, and ankle clonus present. The plantar response was equivocal. The abdominal reflexes were diminished. There was no apparent sensory loss. There was retention of urine with overflow and the bladder was distended to above the level of the umbilicus, the bladder being presumably of the automatic type. The prostate felt normal on rectal examination. A soft rubber catheter 
could not be passed beyond the external sphincter. The urine was normal. Serum tests for syphilis were positive and the cerebrospinal fluid showed the following changes :

lymphocytes 25 per cu. mm., protein $120 \mathrm{mg}$. per cent., globulin increased, Lange normal, Wassermann reaction positive.

10 M.U. crystalline penicillin were given, 80,000 units 4-hourly. The bladder was drained by intermittent catheterization until January 4, 1949 when Mr. WillsonPepper performed a supra-pubic cystostomy. On November 5, 1949 his mental condition was unchanged and the cerebrospinal fluid still showed signs of activity. The urine was sterile. A course of procaine penicillin, 1 M.U. daily for 20 days was given. On December 6, 1949 Mr. Willson-Pepper closed the cystostomy and resected the posterior urethra as in the previous cases. After the operation, the patient was able to empty his bladder completely and with a good stream, and vesical sensation was only slightly impaired. Despite the development first of a supra-pubic leak which had to be remedied, and then of an abscess in the abdominal wound which resulted in a urinary fistula necessitating a further operation, the urinary flow remained good and there was no residual urine. When the patient was last seen by Mr. Willson-Pepper in February, 1952, micturition was still satisfactory and complete.

\section{Conclusion}

Transurethral resection of the vesical neck in cord bladder is a simple operation almost entirely free from risk and from unpleasant sequelae. Only in one patient has vesical dysfunction been increased by the operation, and only one (Case 2) developed any complication and that due to an accident. Why the operation should have failed in a few cases is unknown, but it may be that insufficient tissue was removed from the vesical neck (Emmett and Beare, 1941). It is not yet possible to say for how long the beneficial effects of the operation will persist, but there is some evidence that in tabes dorsalis the improvement may be maintained for at least a few years. Transurethral resection of the vesical neck has been followed in the majority of cases of cord bladder by disappearance of symptoms and elimination of, or decrease in, amount of residual urine and there is much to support the opinion of Emmett and his colleagues that cord bladder and especially the cord bladder of tabes dorsalis now presents few therapeutic problems.

\section{Sumnary}

Some of the literature describing the management of cord bladder by transurethral resection of the vesical neck is reviewed and three cases of luetic cord bladder treated in this way are reported.

It gives me great pleasure to express my gratitude to Mr. J. K. Willson-Pepper, F.R.C.S.

\section{REFERENCES}

Denny-Brown, D., and Robertson, E. Graeme (1933a). Brain, $56,149$.

-, (1933b). Ibid., 56, 397.

Emmett, J. L. (1940). J. Urol., Baltimore, 43, 692.

-, and Beare, J. B. (1941). J. Amer. med. Ass., 117, 1930.

—, - - (1948). Ibid., 136, 1093.

-

Evans, J. P. (1941). J. Amer. med. Ass., 117, 1927.

Gowers, W. R. (1892). "Diseases of the Nervous System", 2nd ed., vol I, p. 410. Churchill, London.

Langworthy, O. R., Dees, J. E., and Lewis, L. G. (1936). Amer. J. Syph., 20, 364.

- and Kolb, L. C. (1933). Brain, 56, 371.

Nesbit, R. M., and Gordon, W. G. (1941). J. Amer. med. Ass., $117,1935$.

Starr, M. A. (1907). " Organic and Functional Nervous Diseases ", 2nd ed., p. 292. Baillière, Tindall, and Cox, London.

Thompson, G. J. (1945). Nav. med. Bull., Wash., 45, 207.

一, and Jacobson, C. E. (1943). Amer. J. Surg., 61, 224.

Torres, L. F. (1948). J. Philipp. med. Ass., 24, 155.

Williamson, R. T. (1908). "Diseases of the Spinal Cord", p. 310. Oxford Medical Publications, London. 